\title{
Determination of the Orientation Distribution Function from One Pole-figure
}

\author{
JANOS IMHOF \\ Aluterv-Fki, Budapest, Hungary
}

(Received September 30, 1981)

The values of the orientation distribution function and the data of the pole-figure are correlated by products of particular conditional probabilities. One of the possible approximations of these products are obtained and demonstrated with the help of an example showing how the orientation distribution function can thus be obtained.

\section{INTRODUCTION}

The following is a summary of former work (Imhof, 1982).

The measuring of a pole-figure at discreet points results in a division of it into small regions $R_{k}$, having in their centre the measured points, where $k$ denotes the serial number of the small regions.

An arbitrary orientation $g$ is represented in the basic domain of the Eulerian space by one point with coordinates $\left\{\varphi_{1}, \phi, \varphi_{2}\right\}$ and in the $\{H K L\}$ pole-figure by $n$ pole-points.

If all pole-points of two orientations fall into the same $R_{k 1}, R_{k 2}, \ldots, R_{k n}$ units, the two orientations cannot be distinguished. Performing this examination for all orientations, the basic domain can be divided into classes $C_{j}$, that contain orientations indistinguishable on the basis of pole-figure data. Different classes belong to different combinations of $\left\{\boldsymbol{R}_{\boldsymbol{k} i}\right\}$, thus they are distinguishable.

Figure 1 shows one of the classes $C_{j}$ in the basic domain for cubic crystal structure. The $\{001\}$ pole-points of orientations belonging to the class $C_{j}$ are in the same regions $R_{k 1}, R_{k 2}, R_{k 3}$.

The number, size, and shape of the orientation classes depend on the 


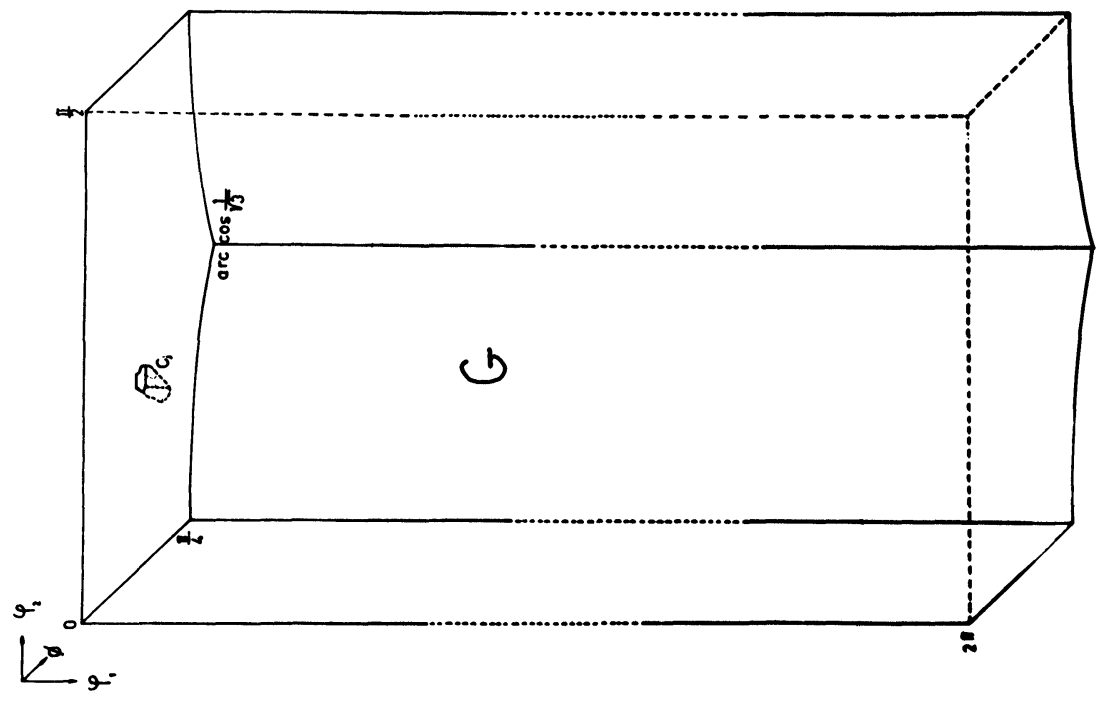

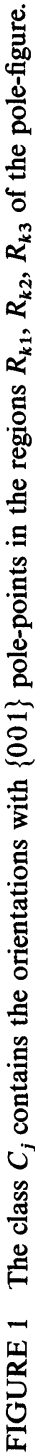


referenced pole-figure. The orientation classes are considered an expression of the character of the pole-figure and of measured pole-figure data structure.

If the angular distance of the measured data are $\Delta \alpha=\Delta \beta=5^{\circ}$, the number of classes in the basic domain are 73500 , using a $\{001\}$ pole-figure.

The value of orientation distribution function inside one class $C_{j}$ is considered by definition to be constant and is denoted by $f\left(C_{j}\right)$. Since different combinations of measured data belong to different classes, in principle there is a possibility of allocating different values of the orientation distribution function to different orientation classes. It means, that in general $f\left(C_{i}\right) \neq f\left(C_{j}\right)$, if $i \neq j$. The unknown function $f\left(C_{j}\right)$ has as many independent variables as the number of classes, in the example above $j_{\max }=73500$.

\section{THE FUNDAMENTAL EQUATION}

Let $D\left(R_{k}\right)$ be the pole density measured in the region $R_{k}$. Let $T_{k}$ denote those particular classes $C_{j}$ which have their $\{H K L\}$ pole points in the region $R_{k} . T_{k}$ is termed an integration tube, consisting of several parts in the basic domain $G$ and these parts are cut at the boundary surfaces of $G$. If $V\left(T_{k}\right)$ denotes the volume of tube $T_{k}$, and $\Delta V\left(C_{j}\right)$ denotes the volume of class $C_{j}$, the form of the fundamental equation is (Imhof, 1982)

$$
D\left(R_{k}\right)=\frac{1}{V\left(T_{k}\right)} \sum_{C_{j} \in T_{k}} f\left(C_{j}\right) \Delta V\left(C_{j}\right)
$$

The left-hand side of Eq. (1) expresses the total pole-density, measured in $R_{k}$, the accuracy of which depends on the measuring method. The right-hand side is the model for this pole-density, having two essential elements: the division of orientation space and the path of integration.

The basic domain of orientation space must be divided into the smallest units distinguishable on the basis of the measured data, but only inside these can we assume that the orientation distribution function is constant. This objective division is realized by classes $C_{j}$. An arbitrary division of the orientation space would falsify from the outset the values of orientation distribution function.

The manner of determination of the path of integration used in this paper, differs from the usual one and corresponds to the division of orientation space. From the classes $C_{j}$, those are selected which give one of the $\{H K L\}$ pole-points in the region $R_{k}$. The collection of these $C_{j}$ constitute the path of integration.

$T_{k}$ corresponds to the double integration path of directions $\mathbf{h}=\{\mathrm{H}, \mathrm{K}, \mathrm{L}\}$ and $-\mathbf{h}$ and $D\left(R_{k}\right)$ corresponds to the $\tilde{P}_{\mathrm{h}}(\mathbf{y})$ in the theory of Matthies (Matthies, 1980). 
The number of equations having the form of Eq. (1), being available to calculate the orientation distribution function is equal to the number of units $R_{k}$. This number is several times less than the number of unknowns i.e. the number of classes $C_{j}$.

\section{THE PRINCIPLE OF SUPERPOSITION}

The terms on the right-hand side of Eq. (1) represent the contributions of classes $C_{j}$ belonging to the integration tube $T_{k}$ to the pole-density measured in $R_{k}$. If one class $C_{j}$ is a common part of $n$ integration tubes, then it gives a contribution in the shape of $f\left(C_{j}\right) \Delta V\left(C_{j}\right) / V\left(T_{k i}\right)$ to units $R_{k i}(k i=k 1, k 2, \ldots$, $k n$ ). These contributions can be found by turns in the equations in the form of (1) used in regions $R_{k i}$. The geometrical fractions $\Delta V\left(C_{j}\right) / V\left(T_{k i}\right)$ are the contributions of isotropic distribution and together they represent an elementary pole-figure. $f\left(C_{j}\right)$ is the superposition coefficient of the elementary pole-figure in the material having a texture.

Let us order the contributions of the same $C_{j}$ into one column, the contributions belonging to the same $R_{k i}$ into one row. Using this procedure for all classes, the equations are obtained in the form of (1) for the regions $R_{k i}$. In the arrangement used, each column represents an elementary pole-figure. Application of the principle of superposition of elementary pole-figures or application of the fundamental equation to all of the measured data are the same.

The principle of superposition of elementary pole-figures is used by vector method. But the author says that the fundamental relation is not valid there (Ruer, p. 6, 1979).

One class $G_{n}$ used in vector method contains a large number of classes $C_{j}$ (Imhof, 1982). The elementary pole-figures corresponding to $G_{n}$ are as a matter of fact not elementary ones.

\section{PROBABILITY QUANTITIES}

Since the number of unknowns is much greater than the number of equations, we try to solve the problem with the help of the theory of probabilities (Rényi, 1962).

The orientations $g$ in the basic domain $G$ are considered as elementary events and the pairs in disjunct classes $C_{j}$ as events. To each event $C_{j}$ is allocated a nonnegative quantity $P\left(C_{j}\right)$ called the probability of the event $C_{j}$. 
According to the definition of the orientation distribution function the probability $P\left(C_{j}\right)$ reads (Imhof, 1982)

$$
P\left(C_{j}\right)=\frac{1}{V(G)} f\left(C_{j}\right) \Delta V\left(C_{j}\right)
$$

$\Delta V\left(C_{j}\right)$ is the sum of orientation elements $\left(d g=\sin \phi \cdot d \phi d \varphi_{1} d \varphi_{2}\right)$ around the orientations $g$, belonging to the class $C_{j}$, and $V(G)=\sum_{C_{j} \in G} \Delta V\left(C_{j}\right)$.

Previously $T_{k}$ denoted the collection of classes, giving one of their $\{H K L\}$ pole-points in $R_{k}$. Each $T_{k}$ is a compound event and the probability of its occurrence is:

$$
P\left(T_{k}\right)=\frac{1}{V(G)} \sum_{C_{j} \subset T_{k}} f\left(C_{j}\right) \Delta V\left(C_{j}\right)
$$

The relationship between $P\left(T_{k}\right)$ and the pole-density $D\left(R_{k}\right)$ comes about through (1) and (3):

where

$$
P\left(T_{k}\right)=D\left(R_{k}\right) V\left(T_{k}\right) / V(G)
$$

$$
V\left(T_{k}\right)=\sum_{c_{j} \in T_{k}} \Delta V\left(C_{j}\right)
$$

Let us consider from orientations $g \in G$ those orientations $g^{\prime}$ which belong to a given integration tube $T_{k 2}$, i.e. $g^{\prime} \in T_{k 2}$. Let $\left\{T_{k 1}\right\}$ denote those series of integration tubes which intersect $T_{k 2}$. Let us denote by $P\left(T_{k 1} \mid T_{k 2}\right)$ the probability of finding an orientation $g^{\prime}$ in $T_{k 1} . P\left(T_{k 1} \mid T_{k 2}\right)$ is called the conditional probability of the compound event $T_{k 1}$ under the condition $T_{k 2}$ and it is defined so that the probability of simultaneous occurrences of $T_{k 1}$ and $T_{k 2}$ (denoted by $P\left(T_{k 1} T_{k 2}\right)$ is divided with the probability of occurrence of $T_{k 2}$ (Rényi, 1962). It is assumed that $P\left(T_{k 2}\right) \neq 0$.

$$
P\left(T_{k 1} \mid T_{k 2}\right)=P\left(T_{k 1} T_{k 2}\right) / P\left(T_{k 2}\right)
$$

The formula (5) can be generalized for optional compound events $T_{k 1}$, $T_{k 2}, \ldots, T_{k n}$ :

$$
\begin{aligned}
P\left(T_{k 1} T_{k 2} \ldots T_{k n}\right)= & P\left(T_{k 1}\right) P\left(T_{k 2} \mid T_{k 1}\right) \times \\
& \times P\left(T_{k 3} \mid T_{k 1} T_{k 2}\right) \ldots P\left(T_{k n} \mid T_{k 1} T_{k 2} \ldots T_{k(n-1)}\right)
\end{aligned}
$$

An example follows.

In the case of the pole-figure $\{001\}$ all classes are intersections of three integration tubes $T_{k 1}, T_{k 2}, T_{k 3}$. Thus the left-hand side of (6) is:

$$
P\left(T_{k 1} T_{k 2} T_{k 3}\right)=P\left(C_{j}\right)=\frac{1}{V(G)} f\left(C_{j}\right) \Delta V\left(C_{j}\right)
$$


The factors on the right-hand side-with a known orientation distribution function-are as follows:

$$
\begin{gathered}
P\left(T_{k 1}\right)=\frac{1}{V(G)} \sum_{C_{j} \subset T_{k 1}} f\left(C_{j}\right) \Delta V\left(C_{j}\right) \\
P\left(T_{k 2} \mid T_{k 1}\right)=\frac{1}{V(G)} \sum_{C_{j} \subset T_{k 1} \cap T_{k 2}} f\left(C_{j}\right) \Delta V\left(C_{j}\right) / \frac{1}{V(G)_{C_{j}}} \sum_{T_{k 1}}^{\prime} f\left(C_{j}\right) \Delta V\left(C_{j}\right) \\
P\left(T_{k 3} \mid T_{k 1} T_{k 2}\right)=\frac{1}{V(G)_{C_{j}} \subset T_{k 1} \cap \sum_{k 2} \cap T_{k 3}} f\left(C_{j}\right) \Delta V\left(C_{j}\right) / \\
\frac{1}{V(G)_{c_{j}} \subset T_{k 1} \cap T_{k 2}} \sum_{\left.\sum_{j}\right) \Delta V\left(C_{j}\right)} f\left(C_{j}\right)
\end{gathered}
$$

With these factors Eq. (6) is transformed into an identity.

From (4) we get

$$
f\left(C_{j}\right)=P\left(T_{k 2} \mid T_{k 1}\right) P\left(T_{k 3} \mid T_{k 1} T_{k 2}\right) \frac{V\left(T_{k 1}\right)}{\Delta V\left(G_{j}\right)} D\left(R_{k 1}\right)
$$

Generally, using $n$ pole-points to identify classes $C_{j}$, i.e. if $C_{j}=T_{k 1} \cap T_{k 2} \ldots$ $\cap T_{k n}$, then

$$
f\left(C_{j}\right)=M_{k i}\left(C_{j}\right) D\left(R_{k i}\right)
$$

where for example

$$
\begin{aligned}
M_{k 1}\left(C_{j}\right)=P\left(T_{k 2} \mid T_{k 1}\right) P\left(T_{k 3} \mid\right. & \left.T_{k 1} T_{k 2}\right) \ldots \times \\
& \times P\left(T_{k n} \mid T_{k 1} T_{k 2} \ldots T_{k(n-1)}\right) V\left(T_{k 1}\right) / \Delta V\left(C_{j}\right)
\end{aligned}
$$

The expression of $M_{k i}\left(C_{j}\right)$ follows from (13) by the cyclical exchange of the letters $k 1, k 2, \ldots, k n$.

If the measured pole-density $D\left(R_{k i}\right)=0$, it follows directly from (12) that $f\left(C_{j}\right)=0$ in all classes $C_{j}$ for which $C_{j} \subset T_{k i}$. The general expression (12) contains the zero-field concept: (Imhof, 1977, 1978) without further ado. In other words, the zero field does not play any favoured role in this method (see Example II).

For the direct determination of the orientation distribution function it is necessary to determine the conditional probabilities contained in the expression of $M_{k i}\left(C_{j}\right)$. On the basis of our present knowledge this is not possible. Therefore we determine the approximate values of $M_{k i}\left(C_{j}\right)$ by iteration. 


\section{APPROXIMATIVE DETERMINATION OF THE ORIENTATION DISTRIBUTION FUNCTION}

It is assumed, that we know the values of the orientation distribution function after the $(N-1)$-th approximation: $f^{(N-1)}\left(C_{j}\right)$. Placing these values in the general expression of $M_{k i}\left(C_{j}\right)$, we very simply arrive at an approximate form for $M_{k i}\left(C_{j}\right)$ :

$$
M_{k i}\left(C_{j}\right)=f^{(N-1)}\left(C_{j}\right) / \frac{1}{V\left(T_{k i}\right)} \sum_{j} \in T_{k i} f^{(N-1)}\left(C_{j}\right) \Delta V\left(C_{j}\right)
$$

With (12):

$$
f_{k i}^{(N)}\left(C_{j}\right)=M_{k i}^{(N)}\left(C_{j}\right) D\left(R_{k i}\right), k i=k 1, k 2, \ldots, k n
$$

We get $n$ different values depending on which of the tubes is taken as basis. To reduce the error of approximation, we use the following procedure.

a) Separate the integration tubes, intersecting each other at class $C_{j}$ $\left(C_{j}=T_{k 1} \cap T_{k 2} \ldots \cap T_{k n}\right)$ and arrange them so, that for the measured poledensities $D\left(R_{k i}\right)$ belonging to the integration tubes $T_{k i}$ the following condition must be fulfilled:

$$
D\left(R_{k 1}\right) \leqslant D\left(R_{k 2}\right) \ldots \leqslant D\left(R_{k n}\right)
$$

b) Starting from the approximation $f^{(N-1)}\left(C_{j}\right)$, we apply relations (14) and (15) first to the tubes $T_{k 1}$ of all classes $C_{j}$. The approximation obtained is $f_{k 1}^{(N)}\left(C_{j}\right)$.

c) Starting from the approximation $f_{k 1}^{(N)}\left(C_{j}\right)$, we apply relations (14) and (15) to the tubes $T_{k 2}$ of all classes $C_{j}$. The approximation obtained is $f_{k 2}^{(N)}\left(C_{j}\right)$.

d) After application of relations (14) and (15) to the tubes $T_{k n}$ of all classes $C_{j}$, we have taken into account all measurement combinations capable of modifying the original values $f^{(N-1)}\left(C_{j}\right)$. Thus we can write $f^{k n(N)}\left(C_{j}\right)=f^{(N)}\left(C_{j}\right)$.

In all cases $f^{(0)}\left(C_{j}\right) \equiv 1$ and, accordingly, we start from the isotropic distribution. The isotropic distribution apparently is not near the texture of the measured sample. But $f^{(1)}\left(C_{j}\right)$ is already a good starting approximation. During the second iteration period we take into account all measurement combinations, capable of modifying the $f^{(1)}\left(C_{j}\right)$ approximation. Thus $N=2$ is sufficient.

Since $M_{k 1}^{(1)}=1$, therefore $f_{k 1}^{(1)}\left(C_{j}\right)=D\left(R_{k 1}\right)$, where $D\left(R_{k 1}\right)$ is the minimum of pole-densities belonging to the class $C_{j}$ (see (16)). This is equivalent to a previously published, but differently deduced, approximation (Imhof, 1977), 


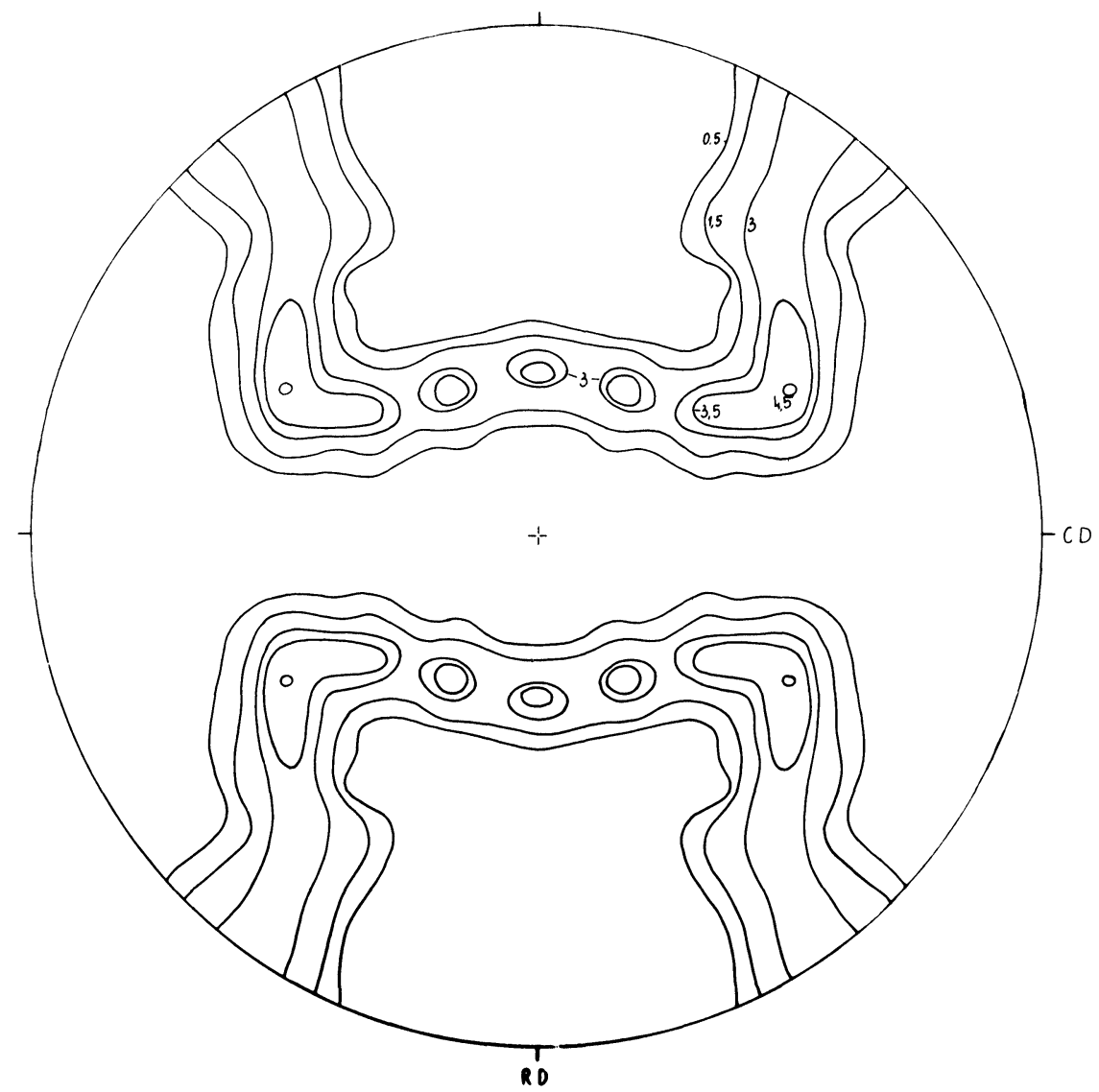

FIGURE 2 Ideal $\left\{\begin{array}{lll}0 & 0 & 1\end{array}\right\}$ pole-figure, which served as the input data for the calculation of orientation distribution function shown in Figure 4.

which, completed with a normalization on the basic domain $G$, gave a good qualitative approximation.

If we continue this procedure so that we always take into account only the tubes $T_{k 1}$, accordingly $f_{k 1}^{(1)}\left(C_{j}\right)=f^{(1)}\left(C_{j}\right), f_{k 1}^{(2)}\left(C_{j}\right)=f^{(2)}\left(C_{j}\right), \ldots$, we get an approximation which is analogous to that of Imhof (1978). Both previous variations have the disadvantage of taking into account only one of the pole-points belonging to one orientation.

\section{RESULTS}

Two examples are given below. 


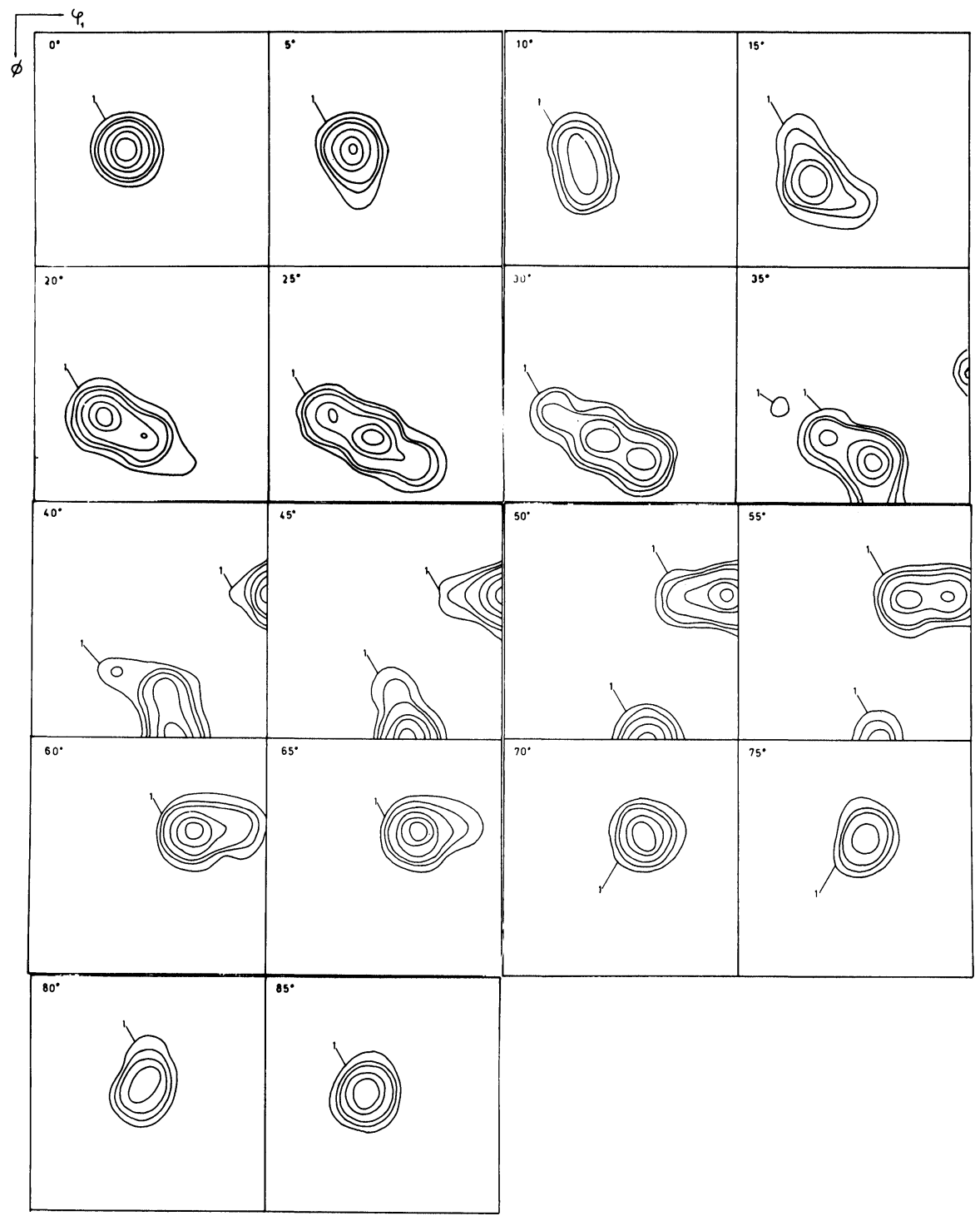

FIGURE 3 Constructed, ideal orientation distribution function from which was calculated the $\left\{\begin{array}{lll}0 & 0 & 1\end{array}\right\}$ pole-figure shown in Figure 2. Level heights: 1, 3, 5, 10, 15, 20, 25.

\section{Example I}

Dr. J. Pospiech and his co-worker, K. Pawlik provided us with a constructed, ideal orientation distribution function and a $\left\{\begin{array}{lll}0 & 0 & 1\end{array}\right\}$ pole-figure calculated 


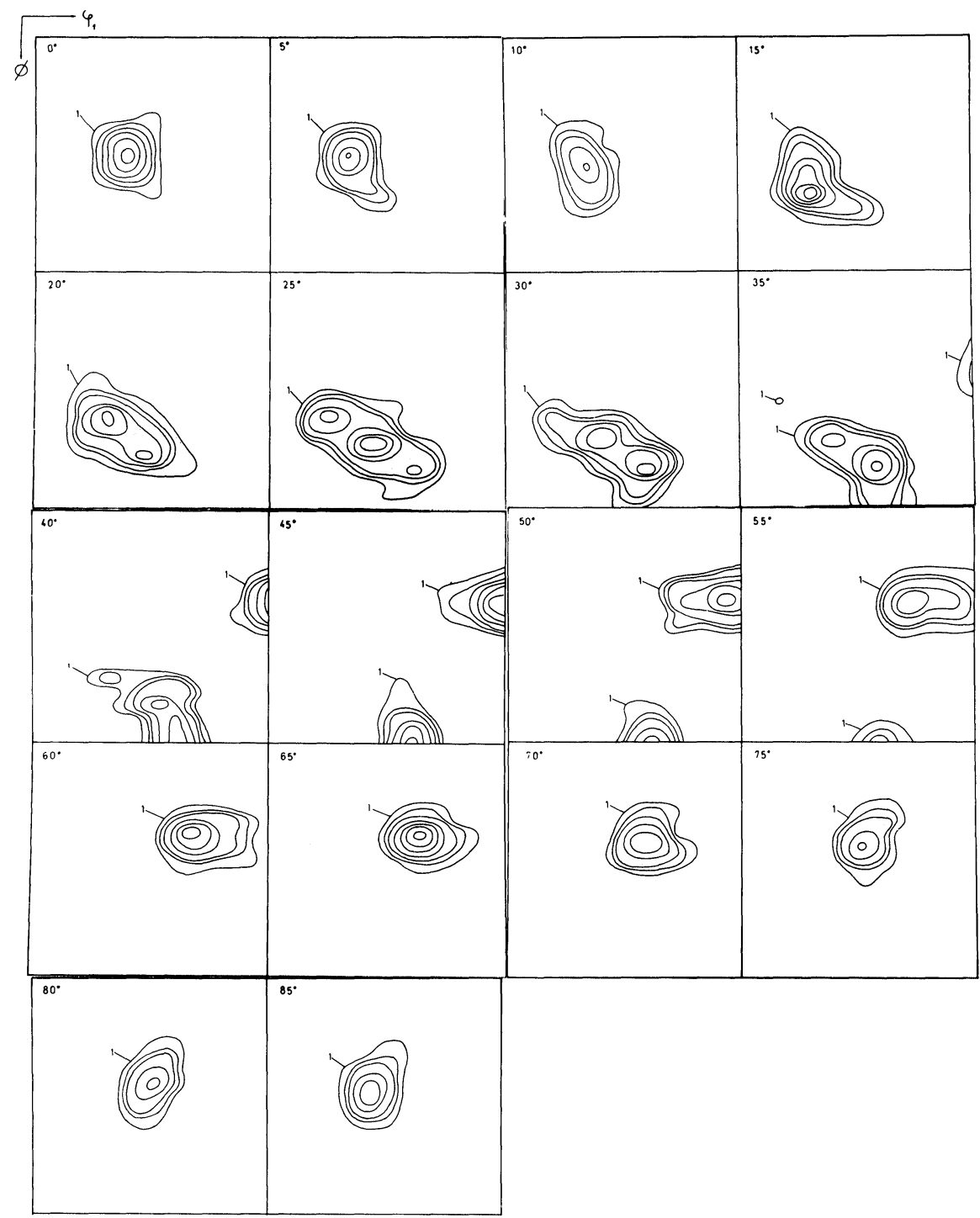

FIGURE 4 The orientation distribution function $f^{(2)}\left(C_{j}\right)$ calculated from the $\{001\}$ pole-figure, shown in Figure 2. Level heights: 1, 3, 5, 10, 15, 20, 25.

from it (see Figures 2 and 3). Since the orientation distribution function falls into symmetrical parts, it is sufficient to represent only the sections shown in Figure 3.

The input data for our evaluation were contained in the $\left\{\begin{array}{lll}0 & 0 & 1\end{array}\right\}$ pole-figure. 


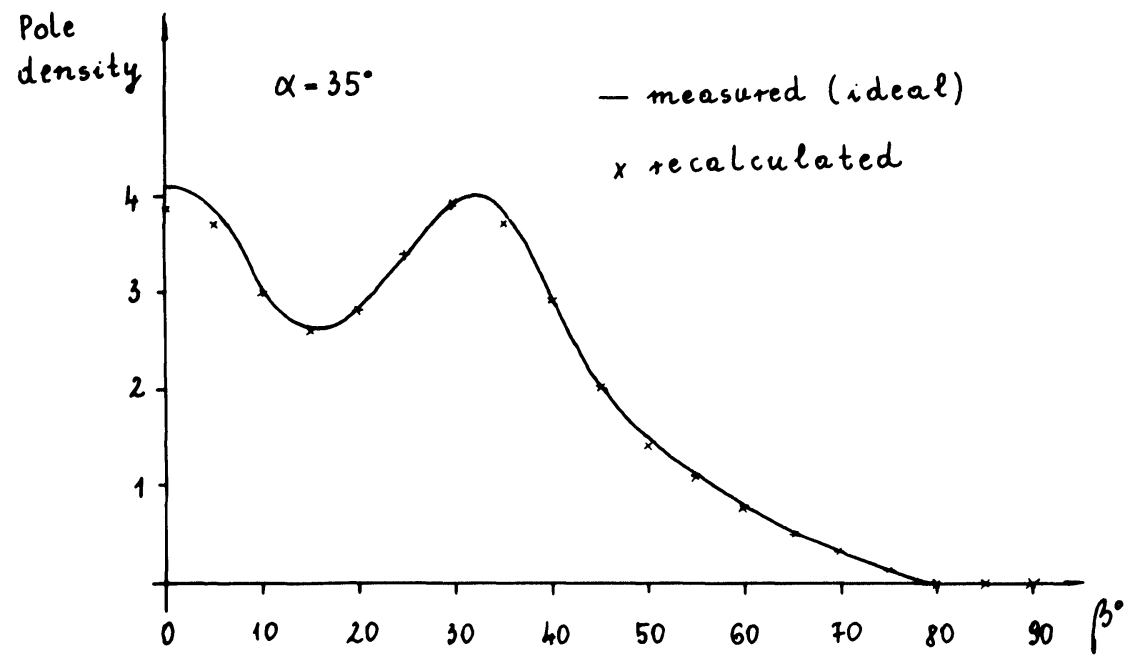

FIGURE 5 Section of the $\{001\}$ pole-figure $D\left(R_{k}\right)$. x: From $f^{(2)}\left(C_{j}\right)$ recalculated points.

From the original quarter pole-figure a complete one (shown in Figure 2) was generated, since the program works as if the pole-figure had no samplesymmetry. The program was worked out in this way because deviations from the ideal sample symmetry are of great importance. Of course, if the starting pole-figure has ideal sample symmetry, as in our case, the orientation distribution function calculated from it must reflect exactly this symmetry.

The number of iterations were $N=2$. The calculated orientation distribution function is shown in Figure 4. The $\{001\}$ pole-figure, recalculated from this function, reproduced the original pole-figure with a sufficient accuracy. For this reason it has not been included. Figure 5 shows a section of the original pole-figure and the recalculated points.

After $N=2$

$$
\frac{1}{V(G)} \sum_{C_{j} \in G} f^{(2)}\left(C_{j}\right) \Delta V\left(C_{j}\right)=1.0012
$$

The ratio of the volume of the zero domain to $V(G)$ was $73 \%$.

\section{Example II}

Let us modify the values of the ideal $f\left(C_{i}\right)$ in the following wayt:

$$
f^{\prime}\left(C_{j}\right)=\left(f\left(C_{j}\right)+A\right) /(1+A)
$$

†Consultation with Dr. S. Matthies. 


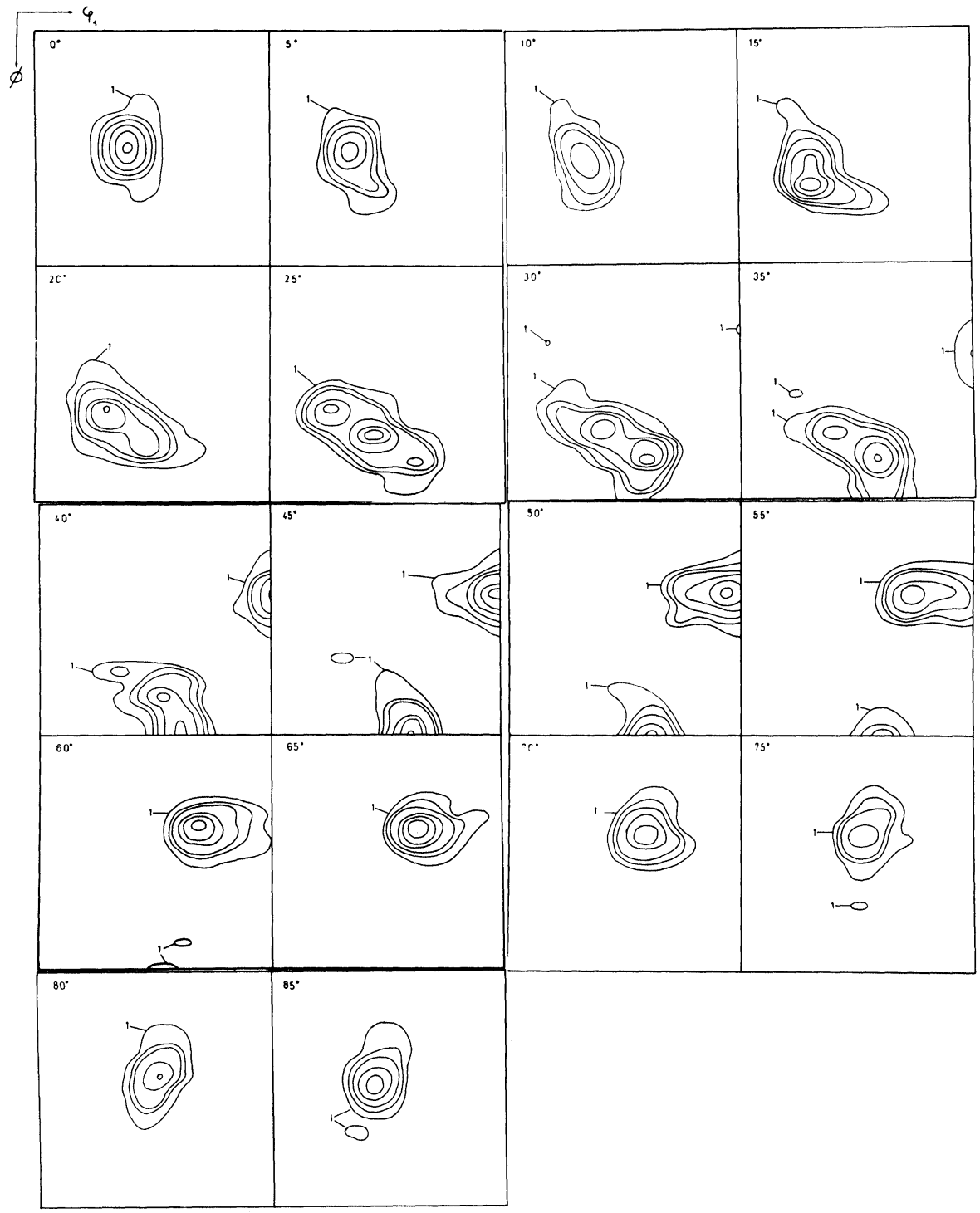

FIGURE 6 The orientation distribution function $f^{\prime}\left(C_{j}\right)$ calculated from the pole-figure $D^{\prime}\left(R_{k}\right)=$ $\left(D\left(R_{k}\right)+0.5\right) /(1+0.5)$. Level heights: 1.02 .33 .77 .010 .313 .717 .0 .

A: Positive constant According to (1) we get a pole-figure with pole-densities

$$
D^{\prime}\left(R_{k}\right)=\left(D\left(R_{k}\right)+A\right) /(1+A)
$$

i.e. we have a pole-figure without zero values. $D\left(R_{k}\right)$ is the ideal pole-figure, 
calculated from ideal $f\left(C_{j}\right)$, (Example I). If we start from pole-figure $D^{\prime}\left(R_{k}\right)$, we must get back $f^{\prime}\left(C_{j}\right)$ approximately. If we draw the level heights

$$
L H^{\prime}=(L H+A) /(1+A)
$$

where $L H$ are the level heights, used in Example I, we must get back, approximately, the same level lines as in Example I, if the method does not depend on the zero values of the pole-figure.

In our example was $\mathrm{A}=0.5$ and $N=2$. The calculated $f^{\prime}\left(C_{j}\right)$ orientation distribution function is shown in Figure 6. The minimal values of this function are 0.33 in principle. The recalculated values were generally between 0.20 and 0.60 at that place. Where $f^{\prime(2)}\left(C_{j}\right)$ reached the level 1 , it was drawn. The comparison of Figure 4 with Figure 6 reinforces our proposal that the method does not essentially depend on the existence of the zero values in the polefigure. It was

$$
\frac{1}{V(G)} \sum_{C_{j} \in G} f^{\varkappa(2)}\left(C_{j}\right) \Delta V\left(C_{j}\right)=0.9995
$$

Figure 7 shows a section of the original pole-figure $D^{\prime}\left(R_{k}\right)$ and the recalculated points from $f^{\prime}\left(C_{j}\right)$.

The work was carried out on an IBM 3031 computer belonging to Hungarian Academy of Sciences. The CPU-time was 1 minute in Example I and 2 minutes in Example II.

\section{Acknowledgements}

A research grant from the Polish Academy of Sciences is gratefully acknowledged. Special thanks are due to Dr. J. Pospiech and Prof. Dr. W. Truszkowski for their contributions, to Dr. S. Matthies for the consultation and to Dr. P. Gangli who provided appropriate computer facilities in Hungary.

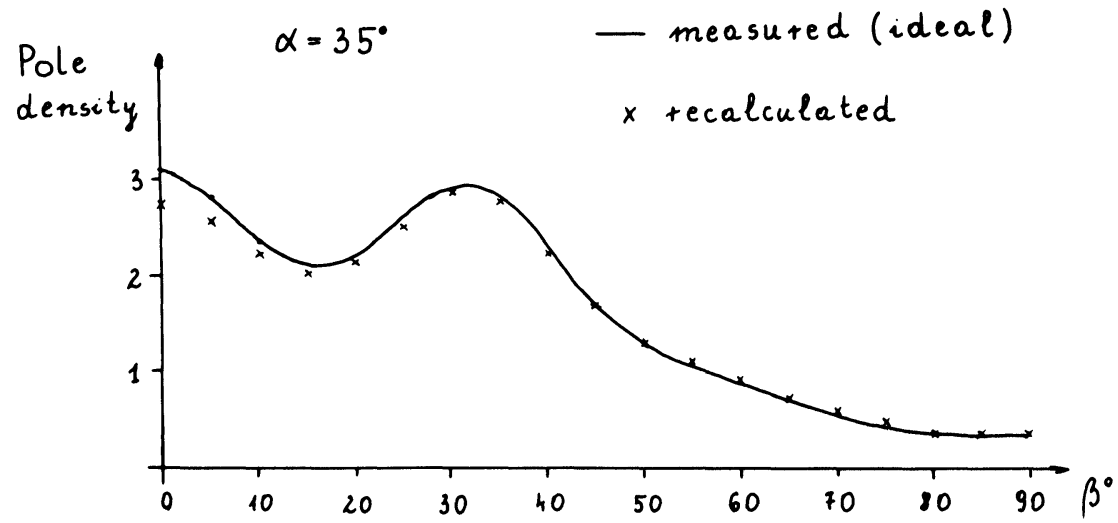

FIGURE 7 Section of the $\{001\}$ pole-figure of $D^{\prime}\left(R_{k}\right)=\left(D\left(R_{n}\right)+0.5\right) /(1+0.5)$. x: From $f^{\prime(2)}\left(C_{j}\right)$ recalculated points. 


\section{References}

Imhof, J. Zeitschrift für Metallkde 68, 38 (1977).

Imhof, J. Textures of Materials I, 149 (1978).

Imhof, J. Textures and Microstructures 4, 189 (1982).

Matthies, S. Kristall und Technik 15, 431 (1980).

Rényi, A. Wahrscheinlichkeitsrechnung. Deutscher Verlag der Wissenschaft (1962).

Ruer, D. The Vector Method, Vol. 14. Summer School, Metz (1979). 